\title{
THE SENSITIVITY OF CLINICAL ISOLATES OF LEISHMANIA FROM PERU AND NEPAL TO MILTEFOSINE
}

\author{
VANESSA YARDLEY,* SIMON L. CROFT, SIMONNE DE DONCKER, JEAN-CLAUDE DUJARDIN, \\ SIDDHARTHA KOIRALA, SUMAN RIJAL, CESAR MIRANDA, ALEJANDRO LLANOS-CUENTAS, AND \\ FRANCOIS CHAPPUIS \\ Department of Infectious and Tropical Diseases, London School of Hygiene \& Tropical Medicine, London, United Kingdom; Prince \\ Leopold Institute of Tropical Medicine, Antwerp, Belgium; B P Koirala Institute of Health Sciences, Dharan, Nepal; Instituto \\ Tropical "Alexander von Humbolt," Lima, Peru; Hôpitaux Universitaires de Genève, Switzerland
}

\begin{abstract}
Clinical isolates of Leishmania, from visceral leishmaniasis (VL) cases in Nepal and from cutaneous leishmaniasis (CL) cases in Peru, were cultured using polymerase chain reaction-restriction fragment length polymorphism (PCR-RFLP) to type species and strain. Promastigotes from 38 isolates, within eight passages from isolation, were used to infect mouse peritoneal macrophage cultures in vitro, and the amastigote sensitivity to miltefosine was determined. The concentration required to kill $50 \%$ of intracellular amastigotes from Nepalese VL isolates, all typed as Leishmania (L.) donovani $(N=24)$ from both $\mathrm{Sb}^{\mathrm{v}}$ responders and nonresponders, ranged from 8.7 to $0.04 \mu \mathrm{g} / \mathrm{mL}$. In contrast, the concentration required to kill $50 \%$ intracellular amastigotes from isolates from Peru, typed as $L$. $(V$.) braziliensis $(N=8)$, was $>30$ to $8.4 \mu \mathrm{g} / \mathrm{mL}$, L.(V.) guyanensis $(N=2)>30$ to $1.9 \mu \mathrm{g} / \mathrm{mL}$, L. $(L$. $)$ mexicana $(N=1)>$ $30 \mu \mathrm{g} / \mathrm{mL}$, and $L$. (V.) lainsoni $(N=4)$ was 3.4 to $1.9 \mu \mathrm{g} / \mathrm{mL}$. This demonstrates a notable difference in the intrinsic sensitivity of Leishmania species to miltefosine in vitro. If this model can be correlated to therapeutic outcome, it may have implications for the interpretation of clinical trials.
\end{abstract}

\section{INTRODUCTION}

The current chemotherapy for leishmaniasis is limited. Pentavalent antimonials have been the recommended drugs for the treatment of both visceral (VL) and cutaneous leishmaniasis (CL) for more than 50 years, but long courses, toxicity, and resistance in India limit their use. New drugs have become available in recent years for the treatment of both $\mathrm{VL}$, including highly efficacious but expensive lipid amphotericin $\mathrm{B}$ formulations, of which AmBisome is the only one widely available. Oral miltefosine has recently been licensed for use in India for the treatment of VL. Topical formulations of paromomycin and oral miltefosine have been used to treat CL. ${ }^{1}$

Miltefosine is an alkylphosphocholine, initially developed as an anticancer agent, that also shows selective activity against Leishmania. ${ }^{2}$ Miltefosine has undergone successful clinical trials for anthroponotic VL in Bihar state, India, with $94 \%$ cure rate, including antimony-resistant cases, ${ }^{3}$ and is currently in Phase IV trials in India and Nepal. This oral drug has demonstrated some activity in the treatment of zoonotic CL in South America ${ }^{4,5}$ where it is currently in Phase III clinical trials. ${ }^{6}$ There are more than 17 species of Leishmania that cause leishmaniasis in human populations; these species have been shown to vary in sensitivity to a range of antileishmanial drugs ${ }^{7}$ including miltefosine. ${ }^{8}$ The current study is the first to evaluate the in vitro anti-leishmanial activity of miltefosine against recently isolated, typed Leishmania spp. from patients in the Old and New Worlds.

\section{MATERIALS AND METHODS}

Ethical clearance was obtained from the ethical committees of the Health Research Council, Kathmandu, Nepal, and Cayetano Heredia University, Lima, Peru. Patients were recruited to the study from November 2002 until the beginning of 2004. Clinical cases of VL in Nepal were recruited at the B

* Address correspondence to Vanessa Yardley, ITD, LSHTM, Keppel Street, London WC1E 7HT, United Kingdom. E-mail: Vanessa. Yardley@lshtm.ac.uk; scroft@dndi.org
P Koirala Institute of Health Sciences, Dharan, Nepal, from a catchment area covering the eastern Terai. Individuals less than 2 years old were excluded from the study. Suspected VL cases, fever for 14 days or longer with splenomegaly, were confirmed by bone marrow aspiration and visual identification of parasites. All patients received $20 \mathrm{mg} \mathrm{Sb}^{\mathrm{V}} \mathrm{kg}^{-1} \mathrm{day}^{-1}$ intramuscularly for 30 days (sodium antimony gluconate; Albert David Ltd, Calcutta, India). Unresponsive patients subsequently received amphotericin B (amphotericin B deoxycholate) treatment.

Cutaneous leishmaniasis patients were recruited at the Instituto Alexander von Humbolt, Lima, Peru, with geographical location noted in the patient data (Table 1). Pregnant women were excluded from the study. Patients presenting with an ulcerated or nodular lesion were considered. Infection by Leishmania was confirmed by direct examination of punch biopsy material with confirmation by parasite culture or PCR. Patients received $20 \mathrm{mg} \mathrm{Sb}{ }^{\mathrm{V}} \mathrm{kg}^{-1} \mathrm{day}^{-1}$ intravenously for 20 days, except for PER069 who received treatment of 30 days. Unresponsive patients received a repeat course of antimonials. If this was again unsuccessful, patients received intravenous amphotericin B (amphotericin B deoxycholate).

Patient material was isolated directly onto $3 \mathrm{~N}^{9}$ blood slopes with a saline/antibiotic overlay and sent to ITG, Antwerp. Parasites were cryopreserved in aliquots and typed within eight passages from isolation. Frozen stocks were sent to LSHTM, London, where the parasites were passaged initially onto $3 \mathrm{~N}$ slopes with M199 + 20\% HIFCS overlay, then into M199 + 20\% HIFCS alone. It was necessary to introduce the use of M199 ${ }^{10}$ to obtain a clean, bulk culture of promastigotes that would be sufficient for further evaluation. There is some evidence that the type of medium can affect the infectivity of the parasite, ${ }^{11}$ however, in this study all isolates were exposed to exactly the same growth conditions, and the work was carried out as close to the isolation as possible. Standard operating procedures were established between partners, which would minimize the effect of this variable between the strains. The parasites were tested for their in vitro sensitivity to miltefosine within eight passages from isolation. 
TABLE 1

Sensitivity of Peruvian cutaneous leishmaniasis isolates to miltefosine

\begin{tabular}{|c|c|c|c|c|c|}
\hline Species & ID & $\begin{array}{c}\mathrm{ED}_{50} \pm \underset{(\mathrm{SEM}}{\mathrm{SEg} \text { miltefosine } / \mathrm{mL}} \\
\left(\mathrm{ED}_{90} \pm \mathrm{SEM}\right)\end{array}$ & $\begin{array}{l}\text { Patient details } \\
\text { (Age; M/F) }\end{array}$ & $\begin{array}{c}\text { Region } \\
\text { (Department) }\end{array}$ & $\begin{array}{l}\text { Clinical response to } \\
\text { antimonial treatment } \mathbb{I}\end{array}$ \\
\hline & PER010 & $>30(>30)$ & & & \\
\hline L. (V.) braziliensis & PER012* & $>30(>30)$ & $32 ; \mathrm{M}$ & Cusco & Nonresponder \\
\hline L. (V.) braziliensis & PER014 & $>30(>30)$ & $14 ; \mathrm{M}$ & Junin & Nonresponder \\
\hline L. (V.) braziliensis & PER067 & $28.3(>30)$ & $23 \mathrm{M}$ & Cusco & Nonresponder \\
\hline L. (V.) braziliensis & PER122 & $21.3 \pm 2.5(19.7 \pm 2.4)$ & $35 ; \mathrm{M}$ & Madre de Dios & Responder \\
\hline L. (V.) braziliensis & PER016 & $>30(>30)$ & $22 ; \mathrm{M}$ & Huanaco & Initial cure \\
\hline L. (V.) braziliensis & PER069† & $22.2(>30)$ & $47 ; \mathrm{M}$ & Madre de Dios & Nonresponder/relapse \\
\hline L. (V.) braziliensis & PER010 & $>30(>30)$ & $22 ; \mathrm{F}$ & Cajamarca & Initial cure \\
\hline L. (V.) guyanensis & PER054 & $>30(>30)$ & $29 ; \mathrm{F}$ & Junin & Unknown $\ddagger$ \\
\hline L. (V.) guyanensis & PER132 & $8.4 \pm 2.22(14.42 \pm 4.3)$ & $65 ; \mathrm{M}$ & Junin & Definite cure \\
\hline L. (V.) lainsoni & PER092 & $3.37 \pm 0.1(4.02 \pm 0.25)$ & $33 ; \mathrm{M}$ & Junin & Initial cure \\
\hline L. (V. lainsoni & PER105 & $1.89 \pm 0.17(3.51 \pm 0.22)$ & $13 ; \mathrm{M}$ & Madre de Dios & Responder \\
\hline L. (V.) lainsoni & PER 127 & $3.1 \pm 0.94(>30)$ & 9; M & San Martin & Responder \\
\hline \multirow[t]{2}{*}{ L. (V.) lainsoni } & PER131 & $2.4 \pm 0.22(4.42 \pm 0.43)$ & $20 ; \mathrm{M}$ & Junin & Initial cure \\
\hline & PER068 & $>30(>30)$ & & & \\
\hline L. (L) mexicana & PER068 & $>30$ & $10 ; \mathrm{M}$ & Ayacucho & Unknown $\ddagger$ \\
\hline L. $(V)$ braziliensis & M2903§ & - & - & & - \\
\hline
\end{tabular}

Late stage promastigotes were used to infect primary isolated mouse peritoneal macrophages at a ratio of 7 promastigotes to 1 macrophage in Labtek 16-well tissue culture well slides (VWR Ltd, Poole, UK), in quadruplicate. Infected cultures were maintained in RPMI 1640 medium (Sigma, Gillingham, UK) with $10 \%$ heat-inactivated fetal calf serum (Harlan SeraLab, Loughborough, UK) at $37^{\circ} \mathrm{C}$ (VL strains) or $34^{\circ} \mathrm{C}$ (CL strains) in a $5 \% \mathrm{CO}_{2} / 95 \%$ air mixture. Twentyfour hours after infection, one slide was methanol-fixed and Giemsa-stained to determine the initial level of infection. Cultures were then exposed to miltefosine (hexadecylphosphocholine, HPC; A G Scientific, San Diego, CA) over a dose range of $30,10,3$, and $1 \mu \mathrm{g} / \mathrm{mL}$, in quadruplicate at each concentration. Higher concentrations were not tested due toxicity to macrophage host cells: at concentrations above 30 $\mu \mathrm{g} / \mathrm{mL}$, macrophages infected are destroyed and the slide cannot be evaluated. After 5 days, all slides were methanolfixed and Giemsa-stained. The percent of infected macrophages in each well was determined by microscopy. From a comparison of counts from treated with untreated cultures, the percent inhibition was calculated by sigmoidal regression analysis (MS xlfit) and $\mathrm{ED}_{50}\left(\mathrm{ED}_{90}\right)$ values determined. Leishmania (V.) braziliensis MHOM/BR/75/M2903 was the reference strain used for Peruvian isolates. $L$. (L.) donovani $\mathrm{MHOM} / \mathrm{ET} / 67 / \mathrm{HU} 3$ was used as the reference strains for $\mathrm{Ne}$ palese isolates. Both strains are known as WHO reference strains, as typed by isoenzyme analysis, and are sensitive to the standard antimonials, sodium stibogluconate and meglumine antimoniate.

Leishmania species typing. In Antwerp, Leishmania species typing was performed by polymerase chain reactionrestriction fragment length polymorphism (PCR-RFLP) analysis of cysteine proteinase $b(\mathrm{cpb})$ and gp63 genes for Nepalese strains and by multi-locus PCR-RFLP for Peruvian strains. Briefly, DNA was extracted from cultivated promastigotes with the QIAmp DNA mini Kit (Qiagen, Hilden, Germany), amplified and digested as reported elsewhere. ${ }^{12-14}$ Restriction patterns were resolved by capillary electrophore- sis (2100 Bioanalyzer system, Agilent Technologies, Karlsruhe, Germany) in a micro-chip device (LabChip 1500, Caliper Technologies, Mountain View, CA) and compared with those of reference strains. Standard strains were also re-typed and confirmed to be $L$. (L.) donovani and $L$. $(V$.) braziliensis.

\section{RESULTS}

The range of activity of miltefosine against South American isolates of cutaneous leishmaniasis is shown in Table 1. L. (V.) lainsoni (PER105) was the most sensitive strain tested with an $\mathrm{ED}_{50}$ value of $1.89 \mu \mathrm{g} / \mathrm{mL}$. L. (V.) braziliensis and $L$. (V.) guyanensis isolates were insensitive to miltefosine over the range tested $(30-0.3 \mu \mathrm{g} / \mathrm{mL})$. The only isolates, for example PER127, which showed sensitivity to miltefosine were typed as $L$. (V.) lainsoni, with $\mathrm{ED}_{50}$ values ranging from 3.37 to 1.89 $\mu \mathrm{g} / \mathrm{mL}$.

The activity of miltefosine against VL isolates from Nepal is shown in Table 2 with $\mathrm{ED}_{50}$ values ranging from less than $0.04 \mu \mathrm{g} / \mathrm{mL}$ to $8 \mu \mathrm{g} / \mathrm{mL}$. Results were similar in repeated experiments. Although the strains were identified as two $L$. (L.) donovani types (I and II, based on restriction pattern differences), ${ }^{12,14,15}$ there was no significant difference in sensitivity to miltefosine between them. The differences seen in the RFLP pattern are useful when tracing the genetic relationships among the strains. L. (L.) donovani HU3 is an Ethiopian strain of VL maintained in passage animals. An Indian reference strain would perhaps have been a more appropriate comparator but was not available to us at the time of this study.

\section{DISCUSSION}

This study supports previous work comparing species sensitivity to miltefosine, which found significant differences between New World and Old World CL and demonstrated sig- 
TABLE 2

Sensitivity of Nepalese visceral leishmaniasis isolates to miltefosine

\begin{tabular}{|c|c|c|c|c|c|}
\hline Species & ID & $\begin{array}{c}\mathrm{ED}_{50} \pm \underset{\left(\mathrm{ED}_{90} \pm \mathrm{SEM}\right)}{\mathrm{SEM}} \mu \mathrm{g} \text { miltefosine } / \mathrm{mL} \\
\end{array}$ & $\begin{array}{l}\text { Patient details } \\
\text { (Age; M/F) }\end{array}$ & $\begin{array}{l}\text { Region } \\
\text { (District)§ }\end{array}$ & $\begin{array}{c}\text { Clinical response } \\
\text { to antimonial treatment } \Phi\end{array}$ \\
\hline \multicolumn{6}{|l|}{ L. donovani (I) } \\
\hline L. donovani (I) & BPK025/0 & $0.5 \pm 0.2(0.9 \pm 0.07)$ & $32 ; \mathrm{M}$ & Sunsari & Definite cure \\
\hline L. donovani (II) & BPK026/0 & $1.9 \pm 0.6(6.9 \pm 0.8)$ & $48 ; \mathrm{M}$ & Bhojpur & Definite cure \\
\hline L. donovani (I) & BPK035/0 & $1.32 \pm 0.09(2.5 \pm 0.21)$ & $9 ; \mathrm{M}$ & Saptari & Nonresponder \\
\hline L. donovani (I) & BPK043/0 & $1.0 \pm 0.04(2.2 \pm 0.1)$ & $10 ; \mathrm{M}$ & Sunsari & Definite cure \\
\hline L. donovani (II) & BPK077/0 & $5.6 \pm 0.7(13.5 \pm 1.5)$ & $40 ; \mathrm{M}$ & Saptari & Nonresponder \\
\hline L. (L.) donovani (I) & BPK080/0 & $1.2 \pm 0.1(3.3 \pm 0.2)$ & $36 ; \mathrm{F}$ & Sunsari & Relapse \\
\hline L. (L.) donovani (I) & BPK085/0 & $5.6 \pm 0.6(10.0 \pm 2.2)$ & $32 ; \mathrm{M}$ & Saptari & Definite cure \\
\hline L. (L.) donovani (I) & BPK087/0 & $5.6 \pm 0.6(10.1 \pm 2.7)$ & $40 ; \mathrm{M}$ & Morang & Definite cure \\
\hline L. (L.) donovani (I) & BPK090/0 & $4.9 \pm 0.2(7.4 \pm 0.5)$ & $46 ; \mathrm{M}$ & Sunsari & Definite cure \\
\hline L. (L.) donovani (I) & BPK091/0 & $1.9 \pm 0.06(6.9 \pm 0.8)$ & $40 ; \mathrm{M}$ & Sunsari & Definite cure \\
\hline L. (L.) donovani (I) & BPK164/1† & $2.2 \pm 0.1(8.2 \pm 1.3)$ & $40 ; \mathrm{M}$ & Dhanusa & Nonresponder \\
\hline L. (L.) donovani (I) & BPK $177 / 0 \dagger$ & $8.1 \pm 0.06(0.9 \pm 0.2)$ & $40 ; \mathrm{M}$ & Dhanusa & Relapse \\
\hline L. (L.) donovani (I) & BPK178/0 & $5.7 \pm 0.7(12.6 \pm 0.6)$ & $45 ; \mathrm{M}$ & Sunsari & Initial cure \\
\hline L. (L.) donovani (I) & BPK190/0 & $0.9 \pm 0.2(7.3 \pm 4.0)$ & $10 ; \mathrm{M}$ & Morang & Nonresponder \\
\hline L. (L.) donovani (I) & BPK191/0 & $2.0 \pm 0.1(5.8 \pm 0.8)$ & $16 ; \mathrm{F}$ & Morang & Initial cure \\
\hline L. (L.) donovani (I) & BPK192/0 & $2.0 \pm 0.2(4.1 \pm 0.5)$ & $7 ; \mathrm{F}$ & Morang & Initial cure \\
\hline L. (L.) donovani (I) & BPK206/0 & $3.9 \pm 1.6(10.2 \pm 3.2)$ & $12 ; \mathrm{M}$ & Morang & Initial cure \\
\hline L. (L.) donovani (I) & BPK208/0末 & $0.04 \pm 0.02(1.7 \pm 0.9)$ & $20 ; \mathrm{F}$ & Morang & Initial cure \\
\hline L. (L.) donovani (I) & BPK212/0 & $3.2 \pm 0.3(6.3 \pm 1.1)$ & $50 ; \mathrm{M}$ & Sunsari & Initial cure \\
\hline L. (L.) donovani (I) & BPK274/0 & $0.85 \pm 0.14(4.0 \pm 0.5)$ & $18 ; \mathrm{F}$ & Sunsari & Initial cure \\
\hline L. (L.) donovani (I) & BPK275/0 & $1.03 \pm 0.04(2.6 \pm 0.2)$ & $12 ; \mathrm{F}$ & Morang & Nonresponder \\
\hline L. (L.) donovani (I) & BPK276/0 & $0.2 \pm 0.1(2.4 \pm 0.7)$ & $10 ; \mathrm{F}$ & Sunsari & Initial cure \\
\hline L. (L.) donovani (I) & BKP278/0 & $2.2 \pm 0.2(8.8 \pm 1.3)$ & $7 ; \mathrm{F}$ & Morang & Initial cure \\
\hline L. (L.) donovani (I) & BPK $279 / 0$ & $0.09 \pm 0.73(1.18 \pm 0.2)$ & $10 ; \mathrm{M}$ & Morang & Initial cure \\
\hline L. (L.) donovani & HU3* & $8.7 \pm 0.6(25.7 \pm 2.6)$ & - & & - \\
\hline
\end{tabular}

$*$ HU3 is WHO reference strain L. donovani MHOM/ET/67/HU3.

$\dagger$ Isolates taken after completion of antimonial treatment.

Pregnant patient treated with amphotericin B.

All districts are on the southeastern border of Nepal.

II Initial cure - resolution of clinical symptoms 6 months after treatment, negative bone marrow aspirate. Currently in follow-up period. Definite cure-complete resolution of clinical signs and symptoms with no evidence of relapse 12 months after treatment, negative bone marrow aspirate.

nificantly different sensitivities between New World species. ${ }^{8}$ This report, one of the few that includes both molecular typing and sensitivity studies of a large number of recent isolates from patients, reflecting results seen in a recent clinical trial of miltefosine against cutaneous leishmaniasis. ${ }^{5}$ That study concluded that miltefosine, at $2.5 \mathrm{mg} \mathrm{kg}^{-1} \mathrm{day}^{-1}$, had comparable efficacy to antimonials when treating presumed $L$. (V.) panamensis in Colombia, less so against presumed L. (V.) braziliensis in Guatemala, but needed further evaluation against more endemic species. Earlier studies have described differences in the sensitivity of species and strains of Leishmania to antimonials, azoles, allopurinol, and paromomycin (see Ref. 7). Six laboratory-maintained isolates of Leishmania have also shown variation in sensitivity to miltefosine. In a 3-day amastigote-macrophage assay, $\mathrm{ED}_{50}$ values ranged from 2.6 to $37.7 \mu \mathrm{M}(1-15.4 \mu \mathrm{g} / \mathrm{mL}), L$. (L.) donovani and L.(L.) aethiopica being the most sensitive species and $L$. (L.) major the least sensitive. ${ }^{8}$ In that study, the $\mathrm{ED}_{50}$ values of $L$. (L.) mexicana and $L$. (V.) panamensis were reported as 6.8-10.1 (2.8$4.1 \mu \mathrm{g} / \mathrm{mL})$ and $10.6 \mu \mathrm{M}(4.3 \mu \mathrm{g} / \mathrm{mL})$, respectively. The results presented in this paper on 38 clinical isolates also indicate that L. (L.) donovani is intrinsically sensitive to miltefosine. In contrast, most of the isolates typed to the $L$. $(V$.) braziliensis complex were insensitive with the exception of those identified as $L$. (V.) lainsoni.

As with in vitro sensitivity to antimonials and other antileishmanial drugs, there appears to be intrinsic variation in species sensitivity to miltefosine. If this is reflected in vivo this has important implications for the evaluation of clinical trials of the drug in Latin America. A dosing regimen of miltefosine at $2.5 \mathrm{mg} \mathrm{kg}^{-1} \mathrm{day}^{-1}$ (150 mg of drug) for 28 days was required to have a significant effect against Colombian $L$. (V.) panamensis but was not as effective against Guatemalan $L$. (V.) braziliensis. ${ }^{5}$ It should be noted that the species were identified by historical data in these studies. The evidence suggests that miltefosine may be a useful drug for the treatment of South American CL, however, species identification should perhaps be a priority before treatment to give the best chance of efficacy and to reduce the possibility of either encouraging resistance to develop, a potential problem when considering anthroponotic transmission, or by re-enforcing intrinsic drug insensitivity, as may be the case in zoonotic foci. Resistance mechanisms to miltefosine been identified in laboratoryinduced Leishmania. ${ }^{16,17}$ It would be interesting to see if these were present in unresponsive "wild-type" parasites.

The utility of the in vitro test when assessing isolate drug sensitivity could be improved by testing parasites pre- and post-treatment, but, to date, the number of samples where this is possible is not significant. Finally, the relevance of in vitro data to clinical outcome must also be considered as the two parameters may not necessarily correlate-confounding factors such as host immunity are absent from these tests. ${ }^{18}$ The data serve to emphasize the extremely complex clinical picture of leishmaniasis.

Received June 29, 2004. Accepted for publication February 11, 2005.

Financial support: This study was funded by the European Commission, LEISHNATDRUG-R ICA4-2000-10109.

Authors' addresses: Vanessa Yardley and Simon Croft, London School of Hygiene \& Tropical Medicine (University of London), De- 
partment of Infectious and Tropical Diseases, Keppel Street, London WC1E 7HT, UK. Telephone: +44 (0)20 7927-2462, Fax: +44 (0)20 7927-2807, E-mail: vanessa.yardley@1shtm.ac.uk, simon.croft@lshtm .ac.uk. Simonne de Doncker and Jean-Claude Dujardin, ITMA, Protozoology Unit, Nationalestraat 155, B-2000 Antwerpen, Belgium. Telephone: +32 3 2476358, Fax: +32 3 2476359, E-mail: SDoncker@ proto.itg.be, jcdujard@itg.be. Cesar Miranda and Alejandro LlanosCuentas, Universidad Peruana Cayetano Heredia, Insituto de Medicine Tropical "Alexander von Humbolt," P.O. Box 4214, Lima 100, Peru. Telephone: +511 4827739, Fax: +511 3820338, E-mail: cesar.miranda@mail.mcgill.ca, allanos@upch.edu.pe. Siddhartha Koirala and Suman Rijal, BP Koirala Institute of Health Sciences, Dharan, Nepal. Telephone: +977 2525555 ext 2052, Fax: +977 25 20251, E-mail: siddharthakoirala2001@yahoo.com, sumanrijal2@ yahoo.com. Francois Chappuis, Travel \& Migration Medicine Unit, Geneva University Hospital, 24 Micheli-du-Crest, 1211 Geneva-14, Switzerland. Telephone: +41 22 3729620, Fax: +41 22 3729626, Email: francois.chappuis@hcuge.ch.

Reprint requests: Vanessa Yardley, ITD, LSHTM, Keppel Street, London WC1E 7HT, UK. Fax: +44 (0)20 7927 2807, E-mail: vanessa.yardley@lshtm.ac.uk.

\section{REFERENCES}

1. Croft SL, Coombs GH, 2003. Leishmaniasis-current chemotherapy and recent advances in the search for novel drugs. Trends Parasitol 19: 502-508.

2. Croft SL, Seifert K, Duchene M, 2003. Antiprotozoal activities of phospholipid analogues. Mol Biochem Parasitol 126: 165-172.

3. Sundar S, Jha TK, Thakur CP, Engel J, Sindermann H, Fischer C, Junge K, Bryceson A, Berman J, 2002. Oral miltefosine for Indian visceral leishmaniasis. $N$ Engl J Med 347: 1739-1746.

4. Soto J, Toledo J, Gutierrez P, Nicholls RS, Padilla J, Engel J, Fischer C, Voss A, Berman J, 2001. Treatment of American cutaneous leishmaniasis with miltefosine, an oral agent. Clin Infect Dis 33: E57-E61.

5. Soto J, Arana BA, Toledo J, Rizzo N, Vega JC, Diaz A, Luz M, Gutierrez P, Arboleda M, Berman JD, Junge K, Engel J, Sindermann H, 2004. Miltefosine for new world cutaneous leishmaniasis. Clin Infect Dis 38: 1266-1272.

6. Burroughs $\mathrm{P}, 2004$. AEterna through its subsidiary Zentaris signs partnership with Roche in Brazil for new treatment of leishmaniasis, a devastating tropical diseases. Available at http:// 217.160.S7.239/en/content/downloads/20040202final.pdf.

7. Croft SL, Yardley V, Kendrick H, 2002. Drug sensitivity of Leishmania species: some unresolved problems. Trans $R$ Soc Trop Med Hyg 96: S127-S129.

8. Escobar P, Matu S, Marques C, Croft SL, 2002. Sensitivities of Leishmania species to hexadecylphophocholine (miltefosine),
ET-18-OCH3 (edelfosine) and amphotericin B. Acta Trop 81: 151-157.

9. Evans DA, 1993. In vitro cultivation and biological cloning of Leishmania. Methods Mol Biol 21: 29-41.

10. Coderre JA, Beverley SM, Schimke RT, Santi DV, 1983. Overproduction of a bifunctional thymidylate synthetase-dihydrofolate reductase and DNA amplification in methotrexateresistant Leishmania tropica. Proc Natl Acad Sci U S A 80: 2132-2136.

11. Dey T, Afrin F, Anam K, Ali N, 2002. Infectivity and virulence of Leishmania donovani promastigotes: a role for media, source, and strain of parasite. J Eukaryot Microbiol 49: 270-274.

12. Garcia AL, Kindt A, Llanos A, Bermudez H, Arevalo J, Banuls AL, Tibayrenc M, de Doncker S, Le Ray D, Dujardin J-C, 2005. American tegumentary leishmaniasis: antigen-gene polymorphism, taxonomy and clinical pleomorphism. Infection, Genetics and Evolution 8: 109-116.

13. Victoir K, De Doncker S, Cabrera L, Alvarez E, Arevalo J, Llanos-Cuentas A, Le Ray D, Dujardin JC, 2003. Direct identification of Leishmania species in biopsies from patients with American tegumentary leishmaniasis. Trans $R$ Soc Trop Med Hyg 97: 80-87.

14. Tintaya KWQ, Ying X, Dedet JP, Rijal D, Bolle XD, Dujardin JC, 2004. Antigen genes for molecular epidemiology of leishmaniasis: polymorphism of cysteine proteinase $b$ and surface metalloprotease gp63 in the Leishmania donovani complex. $J$ Infect Dis 189: 1035-1043.

15. Garcia L, Kindt A, Bermudez H, Llano-Cuentas A, Doncker SD, Arevalo J, Tintaya KWQ, Dujardin J-C, 2004. Culture-independent species typing of neotropical Leishmania for clinical validation of a PCR-based assay targeting heat shock protein 70 genes. J Clin Microbiol 42: 2294-2297.

16. Perez-Victoria JM, Perez-Victoria FJ, Parodi-Talice A, Jimenez IA, Ravelo AG, Castanys S, Gamarro F, 2001. Alkyl-lysophospholipid resistance in multidrug-resistant Leishmania tropica and chemosensitization by a novel P-glycoprotein-like transporter modulator. Antimicrob Agents Chemother 45: 24682474.

17. Seifert K, Matu S, Javier Perez-Victoria F, Castanys S, Gamarro F, Croft SL, 2003. Characterisation of Leishmania donovani promastigotes resistant to hexadecylphosphocholine (miltefosine). Int J Antimicrob Agents 22: 380-387.

18. Aubouy A, Bakary M, Keundjian A, Mbomat B, Makita JR, Migot-Nabias F, Cot M, Le Bras J, Deloron P, 2003. Combination of drug level measurement and parasite genotyping data for improved assessment of amodiaquine and sulfadoxine-pyrimethamine efficacies in treating Plasmodium falciparum malaria in Gabonese children. Antimicrob Agents Chemother 47: 231-237. 